\title{
SEQUENTIALLY COMPACT, FRANKLIN-RAJAGOPALAN SPACES
}

\author{
P. J. NYIKOS ${ }^{1}$ AND J. E. VAUGHAN
}

\begin{abstract}
A locally compact $T_{2}$-space is called a Franklin-Rajagopalan space (or FR-space) provided it has a countable discrete dense subset whose complement is homeomorphic to an ordinal with the order topology. We show that (1) every sequentially compact FR-space $X$ can be identified with a space constructed from a tower $T$ on $\omega(X=X(T))$, and (2) for an ultrafilter $u$ on $\omega$, a sequentially compact FR-space $X(T)$ is not $u$-compact if and only if there exists an ultrafilter $v$ on $\omega$ such that $v \supset T$, and $v$ is below $u$ in the RudinKeisler order on $\omega^{*}$. As one application of these results we show that in certain models of set theory there exists a family $\tau$ of towers such that $|\tau|<2^{\omega}$, and $\prod\{X(T): T \in T\}$ is a product of sequentially compact FR-spaces which is not countably compact (a new solution to the Scarborough-Stone problem). As further applications of these results, we give consistent answers to questions of van Douwen, Stephenson, and Vaughan concerning initially $m$-chain compact and totally initially $m$-compact spaces.
\end{abstract}

1. Introduction. A decreasing tower $\left\{T_{\alpha}: \alpha<\delta\right\}$ on the set of natural numbers $\omega$ (with respect to the mod finite order) is a family of infinite sets $T_{\alpha} \subset \omega$ such that (i) if $\alpha<\beta<\delta$, then $T_{\beta} \subset^{*} T_{\alpha}$ (i.e., $\left(T_{\beta}-T_{\alpha}\right)$ is finite and $\left(T_{\alpha}-T_{\beta}\right)$ is infinite), and (ii) there does not exist an infinite $H \subset \omega$ such that $H \subset^{*} T_{\alpha}$ for all $\alpha<\delta$. For convenience, we assume that $\left(\omega-T_{0}\right)$ is infinite. An ultrafilter $u \in \omega^{*}$ is called a $T$-point provided there exists a decreasing tower $T$ such that $T \subset u$.

For $u \in \beta \omega-\omega$ and a sequence $f: \omega \rightarrow X$, we say that a point $x \in X$ is a $u$-limit point of $f(\operatorname{denoted} x=u$-lim $f)$ provided that for every neighborhood $V$ of $x$ in $X$ we have $f^{-1}(V) \in u$. A space is $u$-compact if every sequence has a $u$-limit (see $\left.\left[\mathbf{V}_{\mathbf{3}}, \S 4\right]\right)$. The main results in our paper are the following theorems.

1.1. THEOREM. For every decreasing tower $T$ on $\omega$ there is an associated sequentially compact, noncompact $F R$-space $X(T)$. Conversely, if $X$ is a sequentially compact, noncompact FR-space, then there exists a tower $T$ such that $X=X(T)$.

1.2. THEOREM. Let $X=X(T)$ be a sequentially compact noncompact $F R$ space and $u \in \omega^{*}$. Then $X$ is not $u$-compact if and only if there exists $v \in \omega^{*}$ such that $v \supset T$ and $v \leq_{R K} u$.

Received by the editors February 6, 1986 and, in revised form, June 12, 1986.

1980 Mathematics Subject Classification (1985 Revision). Primary 54A35, 54D20, 54B10, 54A25, $03 E 35$.

Key words and phrases. Franklin-Rajagopalan space, sequentially compact, countably compact, initially $m$-compact, initially $m$-chain compact, totally initially $m$-compact, strongly $m$-compact, product spaces, $P$-points, $T$-points, Rudin-Keisler order, towers, MA+not-CH $+\diamond\left(c, \omega_{1}-\right.$ limits $)$.

A preprint of this paper was entitled Ordinal extensions of $\omega$ and sequential compactness.

${ }^{1}$ Research supported in part by NSF Grant MCS-8003004. 
The inequality in 1.2 is the Rudin-Keisler (pre)-order on the set of ultrafilters on $\omega$, and $v \leq_{\mathrm{RK}} u$ means that there exists a function $f: \omega \rightarrow \omega$ such that $\bar{f}(u)=$ $\left\{A \subset \omega: f^{-1}(A) \in u\right\}=v$ (see [CN, Chapter 9]).

Our first application of these results is a new, consistent solution to the Scarborough-Stone problem [SSt]: Is every product of sequentially compact spaces countably compact? It is well known ( $\mathrm{cf}$. $\left[\mathbf{V}_{\mathbf{3}}, 4.4\right]$ ) that in order to give a negative solution to the Scarborough-Stone problem, one must find a family $F$ of sequentially compact spaces such that for every $u \in \omega^{*}$ there exists $X \in F$ such that $X$ is not $u$-compact.

1.3. COROLLARY. There exists a family of sequentially compact FR-spaces whose product is not countably compact if and only if for every $u \in \omega^{*}$ there exists $a T$-point $v$ such that $v \leq_{R K} u$.

Balcar, Frankiewicz, and Mills [BFM] constructed a model of set theory in which every $u \in \omega^{*}$ is a $T$-point. Since $u \leq_{\mathrm{RK}} u$ is trivially true, Corollary 1.3 applied in this model shows that FR-spaces can be used to give a consistent negative solution to the problem of Scarborough and Stone (Corollary 1.3 can also be applied in certain models of Hechler $[\mathbf{H}]$ (see §3)). All of the previously known consistent solutions to this problem $\left[\mathbf{R}, \mathbf{R W}, \mathbf{J N W}, \mathbf{V}_{\mathbf{1}}, \mathbf{v D}_{\mathbf{2}}\right]$ are given in models of set theory in which the cardinal equality $\mathfrak{b}=\mathfrak{c}$ holds (in $\left[\mathbf{v D _ { 2 }}\right] \mathfrak{b}=\mathfrak{c}$ is shown sufficient), but in the models in [BFM and $\mathbf{H}$ ] the inequality $\mathfrak{b}<\mathfrak{c}$ holds. This difference in set theory is manifested topologically in the number of sequentially compact spaces needed to have a family of such spaces whose product is not countably compact. In each of the previously known solutions mentioned above, the authors constructed a family of $2^{c}$ sequentially compact spaces whose product is not countably compact, but in some models of Hechler we construct a family of less than $\mathfrak{c}$ sequentially compact spaces whose product is not countably compact (see §3).

We give further applications of our results in $\S 3$ by answering questions raised by van Douwen, Stephenson, and Vaughan. In $\S 4$ we give a few results about $T$-points.

In a preprint of this paper, we raised the obvious question: Is the set-theoretic condition in 1.3 a theorem of ZFC? After seeing our preprint, K. Kunen answered this question in the negative. In a letter of 9 August 1985, he proved the following result: $\mathrm{MA}+\neg \mathrm{CH}+\diamond\left(\mathfrak{c}, \omega_{1}\right.$-limits $)$ imply that there exists a selective, non- $T$-point $u_{0}$ in $\omega^{*}$. Since selective ultrafilters are minimal in the RK-order, the $u_{0}$ in Kunen's theorem has the property that for all $T$-points $v \in \omega^{*}, v \Varangle_{\mathrm{RK}} u_{0}$. It is still not known whether there is a model of $\mathfrak{b}<\mathfrak{c}$ where the condition in 1.3 fails.

2. Proofs of Theorems 1.1 and 1.2. Let $X$ be an FR-space. We will identify the countable dense set of isolated points in $X$ with the set $\omega$, and assume that the remainder $W=X-\omega$ is an ordinal $\delta$ with the order topology. To avoid confusion we also assume that in this context $\delta$ and $\omega$ are disjoint. Of course, different FR-spaces may have different remainders; so the ordinal $\delta$ will vary.

Franklin and Rajagopalan constructed the first FR-space as a quotient of $\beta \omega$. These spaces were also studied by R. Levy $[\mathbf{L}]$ for the case $W=\omega_{1}+1$. We give the set-theoretic version of this construction which is similar to spaces of Mary Ellen Rudin [meR] and E. van Douwen $\left[\mathbf{v D}_{\mathbf{1}}, \mathbf{v D}_{\mathbf{3}}\right.$ ].

\section{ProOF OF THEOREM 1.1. Construction of an FR-space $X(T)$ from a tower} $T$. 
Let $T=\left\{T_{\alpha}: \alpha<\delta\right\}$ be a tower. Let $W$ be the well-ordered space $\delta$ with the order topology, and put $X=\omega \cup W$ (recall that we assume $\omega$ and $W$ are disjoint). The points of $\omega$ are to be isolated. For each $\alpha \in W-\{0\}$ define a basic open neighborhood for each $\beta<\alpha$ and each $F \in[\omega]^{<\omega}$ (= the set of all finite subsets of $\omega)$ by

$$
V(\alpha ; \beta, F)=\{\gamma \in W: \beta<\gamma \leq \alpha\} \cup\left(T_{\beta}-T_{\alpha}\right)-F .
$$

If $\alpha=0 \in W$, define $V(0, F)=\{0\} \cup\left(\omega-T_{0}\right)-F$ for each $F \in[\omega]^{<\omega}$ (recall that we assume that $\left(\omega-T_{0}\right)$ is infinite).

It is straightforward to check that these sets form a base for a topology on $X$, and that $X$ is an FR-space (for more details see $\left[\mathbf{v D}_{3}\right]$ ). In addition, this space is sequentially compact and not compact (since $W$ is a limit ordinal). Thus for every tower $T$, there is a sequentially compact, noncompact FR-space $X(T)$, and this proves the first part of Theorem 1.2. We now prove the second part.

2.1. PROPOSITION. If $X=\omega \cup W$ is a sequentially compact, noncompact $F R$-space, then there exists a tower $T$ on $\omega$ such that $X=X(T)$.

PROOF. For $\alpha \in W$, let $U_{\alpha}$ be a clopen, compact set such that $U_{\alpha} \cap W=[0, \alpha]$. If $\alpha<\beta$, then $U_{\alpha} \subset^{*} U_{\beta}$ since otherwise the infinite set $\left(U_{\alpha}-U_{\beta}\right) \cap \omega$ has a limit point in $\left(U_{\alpha}-U_{\beta}\right) \cap W=\varnothing$. Similarly, if $U_{\alpha} \cap \omega \subset^{*} H$ for all $\alpha \in W$, then $\omega-H$ is finite. Put $T_{\alpha}=\omega-U_{\alpha}$. This set is infinite since $X$ is not compact. Thus, $T=\left\{T_{\alpha}: \alpha \in W\right\}$ is a decreasing tower on $\omega$. We show that $X=X(T)$. By definition, the set underlying the space $X(T)$ is $\omega \cup W=X$. Thus, we have to show that the topologies are the same. A typical basic open neighborhood of $\alpha \neq 0$ in $X(T)$ is of the form $V(\alpha ; \beta, F)=(\beta, \alpha] \cup\left(T_{\beta}-T_{\alpha}\right)-F$, where $\beta<\alpha$ and $F \in[\omega]^{<\omega}$. By definition of $U_{\alpha}$, we see that $V(\alpha ; \beta, F)=\left(U_{\alpha}-U_{\beta}\right)-F$. A similar argument works for $\alpha=0 \in W$. This shows that the topology on $X(T)$ is contained in the topology on $X$. Conversely, if $U$ is open in $X$ and $\alpha \in U \cap W$ $(\alpha \neq 0)$ there exists $\beta<\alpha$ such that $(\beta, \alpha] \subset U$. This implies that $\left(U_{\alpha}-U_{\beta}\right) \subset^{*} U$. Thus, for the obvious $F$,

$$
\alpha \in(\beta, \alpha] \cup\left(T_{\beta}-T_{\alpha}\right)-F \subset U .
$$

The case for $\alpha=0$ is similar and this completes the proof.

If in the proof of 1.1, another choice of $U_{\alpha}$ (say $U_{\alpha}^{\prime}$ ) were made such that $U_{\alpha}^{\prime} \cap W=$ $[0, \alpha]$, we see that $\left(U_{\alpha}-U_{\alpha}^{\prime}\right) \cup\left(U_{\alpha}^{\prime}-U_{\alpha}\right)$ is finite. Hence the towers $\left\{U_{\alpha}: \alpha \in W\right\}$ and $\left\{U_{\alpha}^{\prime}: \alpha \in W\right\}$ are the same in the mod finite order. In this sense we may refer to the tower $T$ associated with a given sequentially compact, noncompact FR-space $X$, and write $X=X(T)$.

ProOF OF THEOREM 1.2. First we assume that $u, v \in \omega^{*}, f \in{ }^{\omega} \omega, \bar{f}(u)=v$, and $v \supset T$. We show that $X(T)$ is not $u$-compact. Let $g$ be $f$ regarded as a function from $\omega$ into $X(T)$. For each $T_{\alpha} \in T$,

$$
g^{-1}\left([0, \alpha] \cup\left(\omega-T_{\alpha}\right)\right)=g^{-1}\left(\omega-T_{\alpha}\right)=f^{-1}\left(\omega-T_{\alpha}\right) \notin u ;
$$

so $g$ has no $u$-limit in $X(T)$. Conversely, assume that $X(T)=\omega \cup W$ is not $u$ compact; say $g: \omega \rightarrow X(T)$ has no $u$-limit in $X(T)$. Define

$$
f(n)= \begin{cases}g(n) & \text { if } g(n) \in \omega, \\ 0 \in \omega & \text { if } g(n) \in W .\end{cases}
$$


We need to show that $f^{-1}\left(T_{\alpha}\right) \in u$ for ail $T_{\alpha} \in T$. For each $T_{\alpha} \in T$, let $K_{\alpha}=$ $\left([0, \alpha] \cup\left(\omega-T_{\alpha}\right)\right) \cup \operatorname{cl}_{X(T)}(g(\omega) \cap W)$. Since $K_{\alpha}$ is compact, $g^{-1}\left(K_{\alpha}\right) \notin u$. Thus $f^{-1}\left(\omega-T_{\alpha}\right) \notin u$ since $f^{-1}\left(\omega-T_{\alpha}\right) \subset g^{-1}\left(K_{\alpha}\right)$. Thus $f^{-1}\left(T_{\alpha}\right) \in u$.

\section{Models in which every $u \in \omega^{*}$ is a $T$-point.}

3.1. Definition. For $f, g \in{ }^{\omega} \omega$ we define $f \leq^{*} g$ provided there exists $N \in \omega$ such that for all $n \geq N, f(n) \leq g(n)$. It is well known that every countable subset of $\omega_{\omega} \omega$ has an upper bound in this mod finite order.

The following lemma expresses in different terminology the technique used by Balcar, Frankiewicz, and Mills to prove the consistency of the statement that every $u \in \omega^{*}$ is a $T$-point.

3.2. LEMMA. Let $\kappa, \lambda$ be cardinals with $\kappa>\operatorname{cf}(\lambda)$. If there exist unbounded chains $\left\{f_{\alpha}: \alpha<\lambda\right\}$ and $\left\{g_{\alpha}: \alpha<\kappa\right\}$ in ${ }^{\omega} \omega$ (with the mod finite order), then there exist a family of $\kappa$ towers each indexed by $\lambda$, and a family of $\lambda$ towers each indexed by $\kappa$, such that every $u \in \omega^{*}$ contains one of these towers. In particular every $u \in \omega^{*}$ is a $T$-point.

In [BFM] it was shown that such pairs of unbounded families can be found by adding $\aleph_{1}$ Cohen reals to a model of MA+not- $\mathrm{CH}$. While this is of independent interest, there is a more flexible way to get such unbounded families of functionsjust call on the following theorem.

3.3. THEOREM (HECHLER $[\mathbf{H}]$ ). If $M$ is a countable standard model of $Z F C$ and $\langle P, \leq\rangle$ is a poset in $M$, and if in $M$ the following hold: (i) the cardinality of $P$ is no more than $2^{\aleph_{0}}$, and (ii) every countable subset of $P$ has an upper bound in $P$, then there is a forcing extension $N$ of $M$ which preserves cofinalities (hence cardinals) and for which $\left(2^{\kappa}\right)_{M}=\left(2^{\kappa}\right)_{N}$ for every cardinal $\kappa$ in $M$, and such that in $N,\langle P, \leq\rangle$ is order isomorphic to a dominating (i.e., cofinal) subset of ${ }^{\omega} \omega$ with the mod finite order.

Hechler pointed out $[\mathbf{H}$, Theorem 8.5] that if we apply this theorem to $P=\kappa \times \lambda$ with the usual product order (i.e., $(\alpha, \beta) \leq(\gamma, \delta)$ iff $\alpha \leq \gamma, \beta \leq \delta$ ), then $P$, and hence also ${ }^{\omega} \omega$ in some model $N$, has an unbounded chain isomorphic to $\lambda$ and another unbounded chain isomorphic to $\kappa$.

3.4. Definition. A space $X$ is initially $m$-chain compact (where $m$ is an infinite cardinal number) if every transfinite sequence $f: n \rightarrow X$ has a convergent (cofinal) subsequence for all infinite $n \leq m$ (see $\left[\mathbf{V}_{\mathbf{2}}\right]$ ). In this terminology, a sequentially compact space is another name for an initially $\omega$-chain compact space. A space $X$ is called initially $m$-compact, provided every open cover of cardinality $\leq m$ has a finite subcover, strongly $m$-compact if every filter base of cardinality $\leq m$ traces on a compact set, and totally initially $m$-compact if for every filter base $\mathcal{F}$ on $X$ with $|\mathcal{F}| \leq m$, there exists a filter base $\mathcal{G}$ on $X$ such that $|\mathcal{G}| \leq m, \mathcal{G}$ is finer than $\mathcal{F}$, and every ultrafilter finer than $\mathcal{G}$ converges to a point of $X$.

Clearly, strong $m$-compactness $\Rightarrow$ total initial $m$-compactness $\Rightarrow$ initial $m$-compactness. Further, if $X=\omega \cup \delta$ is a sequentially compact FR-space, then for every $m<\operatorname{cf}(\delta), X$ is initially $m$-chain compact (this is obvious) and strongly $m$-compact (since every filter base $\mathcal{F}$ on $X$ with $|\mathcal{F}| \leq m$ traces on a compact set of the form $[0, \alpha] \cup\left(\omega-T_{\alpha}\right)$ for some $\left.\alpha<\delta\right)$. 
3.5. COROLLARY. Let $m$ be an infinite cardinal number. It is consistent that $\mathfrak{c}$ be "arbitrarily large" with $m^{++} \leq \mathfrak{c}$, and that there exists a family of $m^{++}$initially $m$-chain compact, strongly m-compact FR-spaces whose product is not countably compact.

ProOF. Take $\kappa=m^{+}, \lambda=m^{++}$, and $P=\lambda \times \kappa$ with the product order. By 3.3 and 3.2 it is consistent that there exist a family $\mathcal{F}$ of $\kappa$ towers indexed by $\lambda$ and a family $\mathcal{G}$ of $\lambda$ towers indexed by $\kappa$ such that every $u \in \omega^{*}$ contains one of these towers. For each $T \in \mathcal{F}, X(T)=\omega \cup m^{++}$and for each $T \in \mathcal{G}$, $X(T)=\omega \cup m^{+}$. In either case, $X(T)$ satisfies both compactness-like properties, and $\prod\{x(T): T \in \mathcal{F} \cup \mathcal{G}\}$ is not countably compact.

This gives consistent answers to two questions raised by Vaughan in $\left[\mathbf{V}_{\mathbf{2}}\right]$. One question asked for examples of initially $m$-chain compact spaces, not $m$-bounded for $m>\omega$. As noted above it is consistent to have FR-spaces $X=\omega \cup W$ with $W$ homeomorphic to $m^{+} \geq \omega_{1}$; so it is consistent to have initially $m$-chain compact spaces which are not $m$-bounded nor even $\omega$-bounded (since $X$ is separable, but not compact). The second question asked if initial $m$-compactness is productive for $m \geq \omega_{1}$. By the preceding corollary, it is consistent that a product of $m^{++} \mathrm{FR}$ spaces can fail to be initially $m$-compact or even countably compact. This question was answered earlier by E. van Douwen who used GCH to construct two initially $m$-compact spaces whose product is not initially $m$-compact $\left[\mathbf{v D}_{\mathbf{3}}\right]$. Our example is different since FR-spaces are initially $m$-chain compact, and it is known that for any $m \geq \omega_{1}$ the product of two (or even $\omega_{1}$ ) initially $m$-chain compact spaces is initially $m$-compact $\left[\mathbf{V}_{\mathbf{2}}\right.$, Theorem 1$]$. Corollary 3.5 also answers consistently three questions raised by R. M. Stephenson, Jr., concerning products of totally initially $m$-compact spaces (see [St, pp. 624, 625]).

We can also answer two questions of van Douwen raised in Topology Proceedings (3 (1978), 532). In Problem (22), he asked: Is initial $m$-compactness productive if $m$ is singular? The answer was known to be "yes" under GCH (see [SS and SV]). Corollary 4.5 shows that the answer is "no" under certain set-theoretic assumptions: Let $m=\aleph_{\omega}, \kappa=m^{+}$, and $\lambda=m^{++}=\mathfrak{c}$ in the ground model. Put $P=\kappa \times \lambda$, and proceed as above. This also answers another related question of van Douwen in Problem (21). For other examples, including cardinals greater than $\mathfrak{c}$, see $\left[\mathbf{v D _ { 2 }}\right.$ ].

The next result shows, among other things, that in a single model of set theory, one can have a variety of families of sequentially compact, FR-spaces with which to answer the question of Scarborough and Stone (in the negative).

3.6. THEOREM. It is consistent that $\mathfrak{c}$ be "arbitrarily large" and that for each infinite cardinal $m$ with $m^{++} \leq \mathfrak{c}$, there exists a family of $m^{++}$initially $m$-chain compact FR-spaces whose product is not countably compact.

ProOF. Given any aleph, $\aleph_{\alpha}$, let $M$ be a model in which $\mathfrak{c}=\aleph_{\beta} \geq \aleph_{\alpha}$, and let $P$ be the $\Sigma$-product of $\left\{\omega_{\alpha}: 0<\alpha \leq \beta, \operatorname{cf}(\alpha) \neq \omega\right\}$, i.e. the set of all points in the product $\prod\left\{\omega_{\alpha}: 0<\alpha \leq \beta, \operatorname{cf}(\alpha) \neq \omega\right\}$ with at most countably many nonzero coordinates, and give $P$ the product order. Then for every $\alpha \leq \beta, P$ has an unbounded chain of cardinality $\omega_{\alpha}$ (e.g., take the set of all functions which are 0 everywhere except at $\alpha$ ). Apply Hechler's theorem (3.3) and Lemma 3.2 to get the required model. 
Using a technique of Z. Frolik (see [ $\mathbf{V}_{\mathbf{3}}$, Example 4.13]) we can also prove

3.7. COROLLARY. It is consistent that there exists a sequentially compact space $Z$ such that the product space $Z^{\omega_{2}}$ is not sequentially compact, and $\mathfrak{c}$ can be arbitrarily large.

4. Remarks about $T$-points. Using the following known technique, we give some rather easy proofs to several basic results about $T$-points.

4.1. Definition ( $\operatorname{SeE}\left[\mathbf{C N}\right.$, p. 156]). For $p, q \in \omega^{*}$, define $p \cdot q$ by

$$
p \cdot q=\{A \subset \omega \times \omega:\{n \in \omega:\{m \in \omega:\langle n, m\rangle \in A\} \in q\} \in p\} .
$$

It is known that $p \cdot q$ is an ultrafilter on $\omega \times \omega$ containing all sets of the form $P \times Q$ for $P \in p$ and $Q \in q$. Let $\pi_{1}$ and $\pi_{2}$ denote the usual projection maps from $\omega \times \omega$ onto $\omega$.

4.2. THEOREM. Let $p, q \in \omega^{*}$. Then $p \cdot q$ is a T-point if and only if $q$ is a T-point.

PROOF. First we prove that if $p \cdot q$ is a $T$-point, then so is $q$. Suppose that $\left\{T_{\alpha}: \alpha<\delta\right\}$ is a decreasing tower contained in $p \cdot q$. Put $S_{i}=\{\alpha<\delta:\{n \in$ $\left.\left.\omega:\langle i, n\rangle \in T_{\alpha}\right\} \in q\right\}$ for every $i \in \omega$. Since $\delta=\bigcup\left\{S_{i}: i \in \omega\right\}$, some $S_{i}$ is cofinal in $\delta$. Then $\left\{T: \alpha \in S_{i}\right\}$ is also a tower in $p \cdot q$. Put $S_{\alpha}=\left\{n \in \omega:\langle i, n\rangle \in T_{\alpha}\right\}$ for all $\alpha \in S_{i}$. Then $S_{\alpha} \in q$ by definition and it is easy to see that $\left\{S_{\alpha}: \alpha \in S\right\}$ is a decreasing tower.

Next we prove the converse. Suppose that $\left\{S_{\alpha}: \alpha<\delta\right\}$ is a decreasing tower in $q$. Of course $\left\{\pi_{2}^{-1}\left(S_{\alpha}\right): \alpha<\delta\right\}$ is not a mod finite tower, so we cut it down to the following set in $p \cdot q$ :

$$
X=\{\langle n, m\rangle: n \leq m\}
$$

Put $T_{\alpha}=\pi_{2}^{-1}\left(S_{\alpha}\right) \cap X$ for all $\alpha<\delta$. First note that $T_{\alpha} \in p \cdot q$ since

$$
\left\{n \in \omega:(\{n\} \times \omega) \cap T_{\alpha} \in q\right\}=\omega \in p .
$$

It is easy to check that $\left\{T_{\alpha}: \alpha<\delta\right\}$ is a decreasing tower.

We now list some consequences of Theorem 4.2.

4.3. COROLLARY. There exists a decreasing tower which is contained in $2^{c}$ ultrafilters. In particular, there exist $2^{\mathfrak{c}} T$-points.

ProOF. Let $q$ be any $T$-point, and $\left\{S_{\alpha}: \alpha<\delta\right\}$ a decreasing tower contained in $q$ and $\left\{T_{\alpha}: \alpha<\delta\right\}$ the tower defined on $\omega \times \omega$ from $\left\{S_{\alpha}: \alpha<\delta\right\}$ in the proof of Theorem 2.3. As shown in that proof, for every $p \in \omega^{*}$, the ultrafilter $p \cdot q$ contains the tower $\left\{T_{\alpha}: \alpha<\beta\right\}$. The result follows from the obvious fact that $p \neq p^{\prime}$ implies $p \cdot q \neq p^{\prime} \cdot q$ (see [CN, 7.21]).

Since $p \cdot q$ is never a $P$-point, we have

\subsection{COROLlaRY. There exists a T-point which is not a P-point.}

It is easy to see that the continuum hypothesis (or more generally " $s=\omega_{1}$ "; see $\left.\left[\mathbf{v D}_{2}\right]\right)$ implies that every $P$-point is a $T$-point. In a preprint of this paper we asked if it is consistent to have a $P$-point which is not a $T$-point. Kunen also answered this question. His (consistent) selective, non- $T$-point $u_{0}$ is (under MA) a $P$-point. 
4.5. COROLlaRY. For every $p \in \omega^{*}$, there exists a family of $T$-points $A \subset \omega^{*}$ such that $A$ has cardinality $2^{\mathfrak{c}}$, and for every $a \in A$, we have $\operatorname{type}(p) \leq_{R K} \operatorname{type}(a)$.

ProOF. From 4.3, we know there are $2^{\mathfrak{c}} T$-points $a$, and that $p \cdot a$ is a $T$-point for each such $a$. The result follows from the known facts that $\bar{\pi}_{1}(p \cdot a)=p$, and $a \neq a^{\prime}$ implies $p \cdot a \neq p \cdot a^{\prime}$ (see [CN, 7.21]).

4.6. COROLLARY. If there exists a non-T-point, then there exists a non-Tpoint $u$ and $a T$-point $a$ with type $(a) \leq_{R K}$ type $(u)$.

4.7. COROLLARY. If there exists one non-T-point, then there exist $2^{\mathfrak{c}}$ non-Tpoints.

\section{REFERENCES}

[BFM] B. Balcar, R. Frankiewicz, and C. Mills, More on nowhere dense closed P-sets, Bull. Acad. Polon. Sci. Ser. Sci. Math. Astronom. Phys. 28 (1980), 295-299.

[B] A. R. Bernstein, A new kind of compactness for topological spaces, Fund. Math. 66 (1970), 185-193.

[CN] W. W. Comfort and S. Negrepontis, The theory of ultrafilters, Springer-Verlag, Berlin and New York, 1974.

[vD1] Eric K. van Douwen, Hausdorff gaps and a nice countably paracompact non-normal space, Topology Proc. 1 (1976), 239-242.

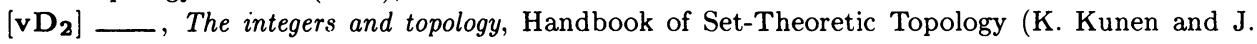
Vaughan, eds.), North-Holland, 1984, pp. 111-167.

[vD $\mathbf{D}_{3}$, The product of two normal initially $\kappa$-compact spaces (to appear).

[FR] S. P. Franklin and M. Rajagopalan, Some examples in topology, Trans. Amer. Math. Soc. 155 (1977), 305-314.

[H] S. H. Heckler, On the existence of certain cofinal subsets of $\omega^{\omega} \omega$, Axiomatic Set Theory (T. J. Jech, eds.), Amer. Math. Soc., Providence, R.I., 1967, pp. 155-173.

[JNW] I. Juhász, Zs. Nagy, and W. Weiss, On countably compact, locally countable spaces, Period. Math. Hungar. 10 (1979), 193-206.

[L] Ronnie Levy, Pseudocompactness and extension of functions in Franklin-Rajagopalan spaces, Topology Appl. 11 (1980), 297-304.

[R] M. Rajagopalan, Some outstanding problems in topology and the $V$-process, Lecture Notes in Math., vol. 540, Springer-Verlag, Berlin and New York, 1975, pp. 501-517.

[RW] M. Rajagopalan and R. Grant Woods, Products of sequentially compact spaces and the $V$ process, Trans. Amer. Math. Soc. 232 (1977), 245-253.

[meR] M. E. Rudin, A technique for constructing examples, Proc. Amer. Math. Soc. 16 (1965), 1320-1323.

[St] R. M. Stephenson, Jr., Initially $\kappa$-compact and related spaces, Handbook of Set-Theoretic Topology (K. Kunen and J. Vaughan, eds.), North-Holland, 1984, pp. 603-632.

[SS] V. Saks and R. M. Stephenson, Jr., Products of m-compact spaces, Proc. Amer. Math. Soc. 28 (1971), 279-288.

[SSt] C. T. Scarborough and A. H. Stone, Products of nearly compact spaces, Trans. Amer. Math. Soc. 124 (1966), 131-147.

[V1] J. E. Vaughan, Products of perfectly normal sequentially compact spaces, J. London Math. Soc. 14, (1975), 517-520.

$\left[\mathbf{V}_{\mathbf{2}}\right] \ldots$, Products of $[a, b]$-chain compact spaces, Houston J. Math. 3 (1977), 569-578.

$\left[\mathbf{V}_{\mathbf{3}}\right] \ldots$, Countably compact and sequentially compact spaces, Handbook of Set-Theoretic Topology (K. Kunen and J. Vaughan, eds.), North-Holland, 1984, pp. 569-602.

DePartment of Mathematics and Statistics, University of South CaRolina, Columbia, South Carolina 29208

Department of Mathematics, University of North Carolina at GreensBORO, GREENSBORO, NORTH CAROLINA 27412 